\title{
Ichthyosis, hepatosplenomegaly, and cerebellar degeneration in a sibship
}

\author{
P S HARPER, R MARKS, P J DYKES, AND I D YOUNG \\ From the Department of Medicine, University Hospital of Wales, Cardiff
}

SUMMARY A sibship is described in which at least two brothers suffer from a unique disorder characterised by ichthyosis, hepatosplenomegaly, and late onset cerebellar ataxia. The clinical features and investigations are described. No metabolic abnormality has been found so far.

Ichthyosis, ataxia, and hepatosplenomegaly may each occur independently in several hereditary disorders. This report describes a sibship where all three have occurred in at least two brothers, and possibly in a third, who is now dead. A fourth brother has only ichthyosis. We suggest that this clinical triad is unique and represents a new disease entity.

\section{Case reports}

The family tree is shown in fig 1 . The findings in the proband, II.1, are described in detail. Other relevant family information follows.

\section{CASE II. 1}

This retired steelworker, born in 1911, has had dry skin since childhood, but was otherwise well until the age of 62 years, when he noted slurring of speech and unsteadiness of gait. Both balance and speech have slowly deteriorated, so that he now walks and talks with difficulty. He also admits to impaired memory for recent events. There is no history of drug ingestion or other serious illness.

Received for publication 10 October 1979

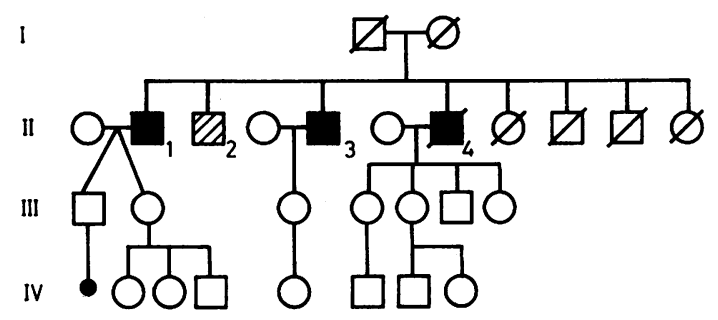

FIG 1 Family pedigree. Shaded subjects show the full syndrome. II.2 shows only ichthyosis.
At recent examination he was plethoric (fig 2) with dry ichthyotic skin most marked on extensor surfaces with flexural sparing (fig 3). His hands showed marked accentuation of the creases (fig 4). Firm hepatosplenomegaly was present with no other stigmata of liver disease. Mentally he showed mild euphoria with evidence of early dementia. Ataxia of both trunk and limb movements and dysarthria were present. There was impairment of upward conjugate gaze but no nystagmus was present and no fundal changes were noted. Reflexes were sluggish with bilateral extensor plantar responses. Power and sensation were normal.

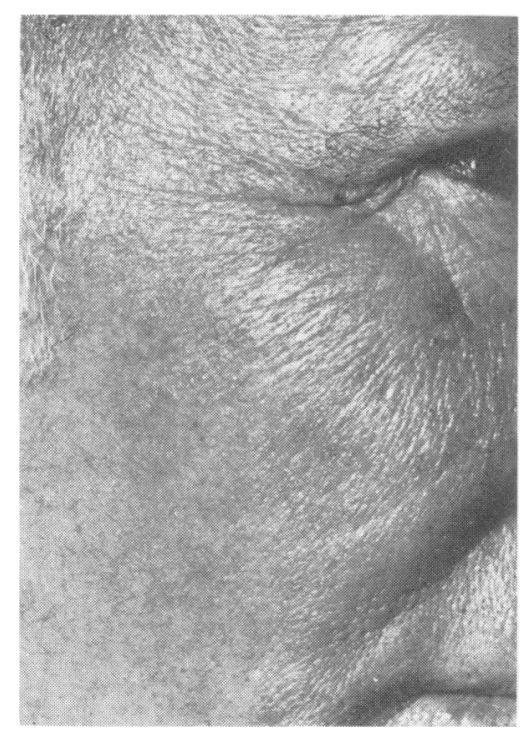

FIG 2 Plethoric dry facial skin observed in 11.1 


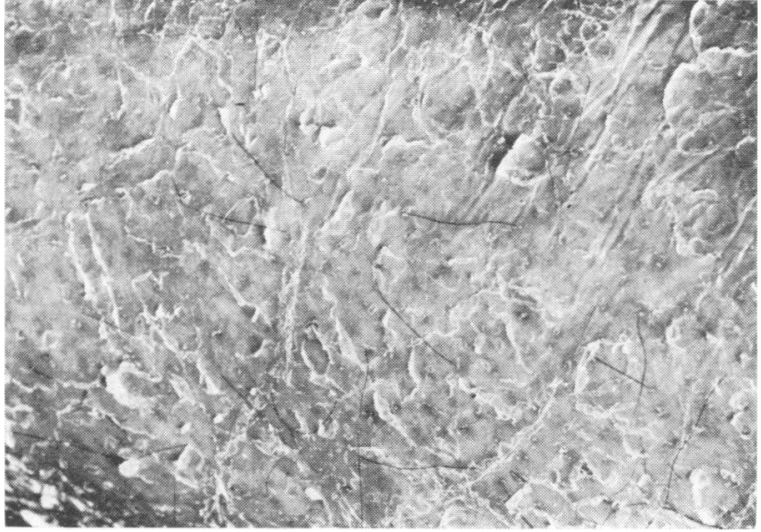

FIG 3 Lower limb ichthyosis in II.1.

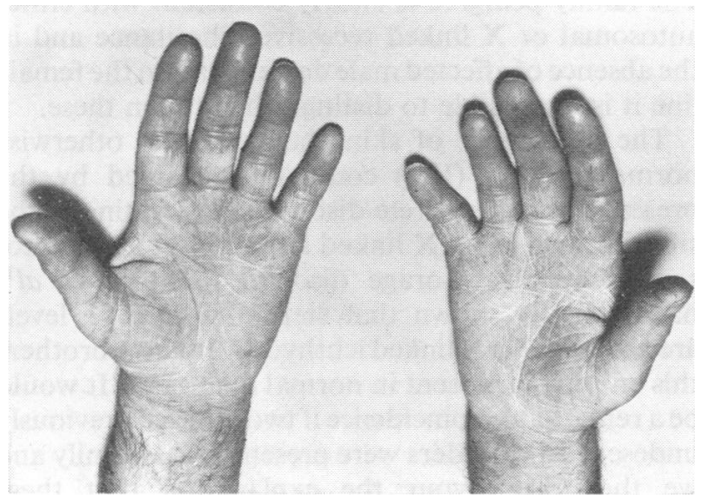

FIG 4 Accentuation of palmar creases in II.1.

CASE II. 2

This subject, born in 1913, has a long history of mild ichthyosis with marked hyperkeratosis of palms and soles (fig 5), but is otherwise symptom free. No hepatosplenomegaly or neurological abnormalities were found on examination.

\section{CASE II. 3}

This ex-coal miner, born in 1915 , with a similar life long history of dry skin affecting mainly the legs and trunk, had a rectal carcinoma removed at the age of 51. One year later, he noticed slurring of speech followed by unsteadiness of gait. These have progressed. Recent examination confirmed the presence of severe cerebellar ataxia with dysarthria, hepatosplenomegaly, mild euphoria with early signs of dementia, and impairment of upward gaze. Skin was dry and ichthyotic as in II.1.

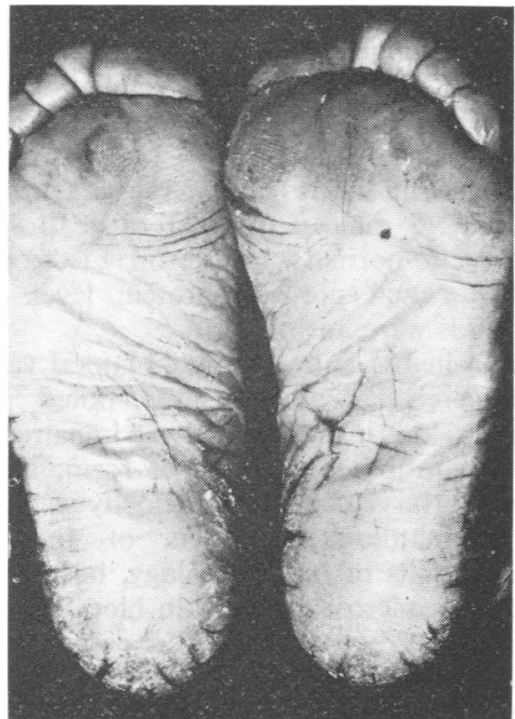

FIG 5 Marked hyperkeratotic changes on feet in II.2.

CASE II. 4

This fourth brother died of cerebral haemorrhage aged 58 years. His hospital notes describe facial plethora with hepatosplenomegaly and ataxia. Mentally he was said to be very slow with slurred speech. No necropsy studies were performed.

The seven offspring of these brothers have been examined and all are free of any signs of the disease. The parents of these brothers I.1 and I.2 died aged 81 and 87 years, respectively, with no evidence of progressive neurological disease. They were unrelated.

\section{Investigations}

In members II. 1 and II.3 the following were normal: routine haematological tests including serum iron and B12 and routine biochemical tests including liver function tests, calcium, phosphate, uric acid, blood and urine amino-acids, blood copper and caeruloplasmin, thyroid function, serum immunoglobulins, and creatine kinase. Biopsies of liver, bone marrow, and sural nerve were also normal with no evidence of storage abnormality. Wasserman reaction and autoimmune antibody screens were negative. No phytanic acid or $5,8,11$, eicosatrienoic acid was detected in the plasma.

The following blood liver and fibroblast enzymes were assayed at the Galton Laboratory in London and were normal; $\mathrm{N}$ acetyl hexosaminidase, $\alpha$-mannosidase, $\alpha$-galactosidase, $\beta$-galactosidase, 
$\alpha$-glucosidase, adenosine deaminase, malate dehydrogenase, phosphogluconate dehydrogenase, phosphoglucomutase, glyoxylase, and acid phosphatase. Fibroblast steroid sulphatase levels were also normal (assayed by PJD in Cardiff).

In II.1 fasting lipids showed raised serum triglycerides of $2.5 \mathrm{mmol} / 1$ (normal range 0.8 to 2.0 $\mathrm{mmol} / \mathrm{l}$ ) with normal cholesterol. Electrophoresis showed a raised pre- $\beta$-lipoprotein level. In II.3 fasting lipids were normal.

CT scan in II.1 showed dilated lateral ventricles. In II.3 cerebellar atrophy was noted. Formal psychometric testing in II.3 showed impaired visualmotor integration, perception of form, and short term recall. Nerve conduction velocity was normal.

The dermatological aspects of this family, including results of histopathology, histochemistry, and electron microscopy on skin biopsies from II.1 and II.3 have been reported elsewhere. ${ }^{1}$ Briefly, marked hyperkeratosis was noted with an increased number of desmosomal complexes in the granular cell layer and adjoining corneal cells. These findings are not typical of any type of ichthyosis yet described.

\section{Discussion}

\section{DIAGNOSTIC ASPECTS}

The clinical features in this sibship strongly suggest a hereditary disorder, although we have been unable to find any specific metabolic abnormality. The presence of hepatosplenomegaly in the affected subjects suggests a storage abnormality, despite the normal findings in liver, bone marrow, and sural nerve. Although central nervous system involvement was initially limited clinically to the cerebellum, there is now evidence of a more generalised cerebral involvement with ophthalmoplegia, dementia, and dilatation of the lateral ventricles on CT scan.

In attempting to classify this disorder several well defined differential diagnoses must be considered. Skin changes are found in many genetic disorders showing multisystem involvement, such as Hartnup's disease where an intermittent photosensitive rash accompanies episodes of cerebellar ataxia. ${ }^{2}$ Several syndromes are recognised in which ichthyosis occurs with neurological signs including spasticity, ${ }^{3}$ oligophrenia, ${ }^{4}$ and polyneuritis with ataxia. ${ }^{5}$ Giroux and Barbeau $^{6}$ have described a French Canadian family where a childhood papulosquamous ichthyosiform eruption preceding the onset of ataxia in later years showed autosomal dominant inheritance. They were unable to trace any records of a similar disorder.

The cerebellar ataxias comprise a large and poorly defined group of disorders showing marked clinical and genetic heterogeneity. Schimke ${ }^{7}$ concluded that

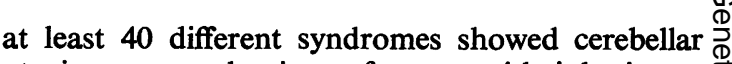
ataxia as a predominant feature, with inheritance $\stackrel{+}{+}$ being either autosomal dominant or recessive or $\mathbf{X} \overrightarrow{\vec{F}}$ linked recessive. External ophthalmoplegia has been $-\frac{7}{0}$ described in some of these families. ${ }^{8}$ Paralysis of $\frac{C}{O}$ upward conjugate gaze is found in at least one form $\overline{\bar{c}}$. of olivopontocerebellar atrophy with onset in late $\vec{\nabla}$ adult life, ${ }^{9}$ and Neville et al ${ }^{10}$ have described vertical $\stackrel{\varrho}{\vartheta}$ ophthalmoplegia in association with neurovisceral os involvement.

In none of these reports do the clinical features correspond sufficiently with those in the subjects of $\vec{\sigma}$ this report to suggest an identical disorder. This factor, coupled with the previously undescribedo ultrastructural findings in the skin, prompts us to $\vec{V}$ suggest that these brothers suffer from a unique $\omega$ hereditary disease.

\section{GENETIC ASPECTS}

The family pedigree is clearly consistent with either autosomal or $\mathrm{X}$ linked recessive inheritance and in $\subseteq$ the absence of affected male descendants in the female $\widehat{\nabla}$ line it is impossible to distinguish between these.

The occurrence of skin changes in an otherwise ${ }^{\circ}$ normal brother (II.2) could be explained by the presence of two discrete disorders segregating in the $O$ sibship, such as an $\mathrm{X}$ linked ichthyosis and an autosomal recessive storage disorder. Shapiro et all1 have recently shown that steroid sulphatase levels $\stackrel{0}{\circ}$ are abnormal in $\mathrm{X}$ linked ichthyosis. In these brothers $\stackrel{\mathbb{2}}{2}$ this enzyme is present in normal quantities. It would $\overrightarrow{\overrightarrow{0}}$ be a remarkable coincidence if two discrete previously 3 undescribed disorders were present in this family and we therefore favour the explanation that these? brothers show variable manifestations of an unidentified pleiotropic metabolic defect, analogous to theco multisystem involvement seen in Refsum's disease ${ }^{5}$ 을 or the Sjögren-Larsson syndrome. ${ }^{3}$

We thank Dr C E C Wells of the University Hospital of Wales for referring the family, Dr A J Franks of 9 Leeds General Infirmary for reporting on the sural $>$ nerve biopsies, and Dr Dallas Swallow of the Galton Laboratory, London for performing the enzyme assays.

This paper was presented at the Clinical Genetics $\tilde{O}^{\circ}$ Society in Oxford, April 1979.

\section{References}

1 Dykes PJ, Marks R, Harper PS. A syndrome of ichthyosis, $\stackrel{\oplus}{?}$ hepatosplenomegaly and cerebellar degeneration. $B r J$ Dermatol 1979;100:585-90.

2 Baron DN, Dent CE, Harris H, Hart EW, Jepson JB. Hereditary pellagra-like skin rash with temporary cere- $\frac{\rho}{\Phi}$ bellar ataxia, constant renal amino-aciduria and other $\stackrel{\mathbb{Q}}{\varrho}$ bizarre biochemical features. Lancet 1956;2:421-8. 
3 Sjögren T, Larsson T. Oligophrenia in combination with congenital ichthyosis and spastic disorders. A clinical and genetic study. Acta Psychiatr Scand (Suppl) 1957;113: 1-112.

4 Passwell JH, Goodman RM, Ziprkowski M, Cohen BE. Congenital ichthyosis, mental retardation, dwarfism and renal impairment: a new syndrome. Clin Genet 1975;8: 59-65.

5 Refsum S, Salomonsen L, Skatvedt M. Heredopathia atactica polyneuritiformis in children. J Pediatr 1949; 35 : 335-43.

- Giroux JM, Barbeau A. Erythrokeratodermia with ataxia. Arch Dermatol 1972;106:183-8.

7 Schimke RN. Adult-onset hereditary cerebellar ataxia and neurosensory deafness. Clin Genet 1974;6:416-21.

${ }^{8}$ Stephens J, Hoover ML, Denst J. On familial ataxia, neural amyotrophy, and their association with progressive external ophthalmoplegia. Brain 1958;81:556-66.

9 Konigsmark BW, Weiner LP. The olivopontocerebellar atrophies: a review. Medicine (Baltimore) 1970;49:227-41.

10 Neville BGR, Lake BD, Stephens R, Sanders MD. A neurovisceral storage disease with vertical supranuclear ophthalmoplegia, and its relationship to Niemann-Pick disease. Brain 1973;96:97-120.

11 Shapiro LJ, Weiss R, Buxmann MM, Vidgoff J, Dimond RL, Roller JA, Wells RS. Enzymatic basis of typical X-linked ichthyosis. Lancet $1978 ; 2: 756-7$.

Requests for reprints to Dr P S Harper, Section of Medical Genetics, Department of Medicine, University Hospital of Wales, Heath Park, Cardiff CF4 $4 \mathrm{XW}$.

\section{Addendum}

Since submitting this paper, patient II.1 died suddenly at home. Necropsy findings were as follows. The lungs showed evidence of acute bronchopneumonia and pulmonary oedema with hypertensive changes in the pulmonary arteries. The heart weighed $500 \mathrm{~g}$ and showed right ventricular hypertrophy. Atheroma was noted in the coronary arteries and distal descending aorta. The liver showed evidence of chronic passive congestion. The spleen weighed $1000 \mathrm{~g}$ and contained large histiocytic cells scattered diffusely throughout (fig 6). Some of these cells showed cytoplasmic vacuoles. No abnormality of this or any other type was noted in bone marrow, testis, thyroid, kidney, or brain, nor were gross changes noted in cerebellum or spinal cord. Further neuropathological studies are in progress.

We wish to thank Dr J N Dearnaley of Nevill Hall Hospital, Abergavenny, for supplying details of necropsy findings.

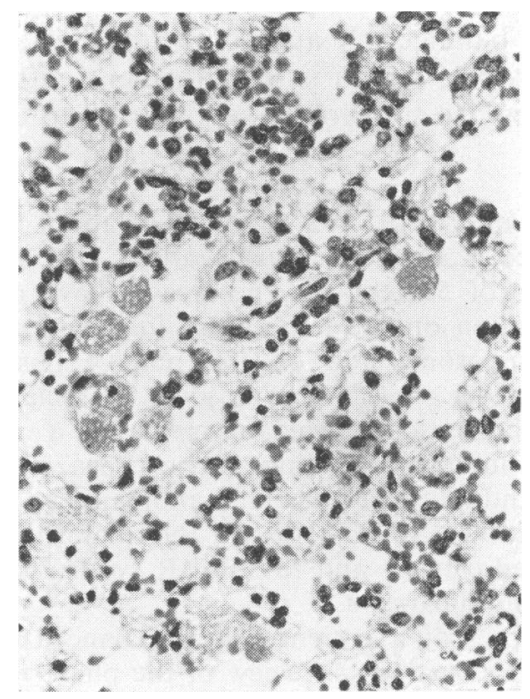

FIG 6 Section of spleen showing foamy histiocytes. 